\title{
Towards Safe Physical Human-Robot Interaction: an Online Optimal Control Scheme
}

\author{
Sholeh Norouzzadeh ${ }^{1}$, Tamara Lorenz ${ }^{1,2}$ and Sandra Hirche ${ }^{1}$
}

\begin{abstract}
Operating in the proximity of humans has been a long-term challenge in robotics research. To achieve this objective, one of the main issues is to ensure safe and comfortable physical human-robot interaction (pHRI). In this paper, we tackle the safety problem at the control level. To ensure operation is within perceived safe zone, we use model predictive control, which finds the optimal control signal online, while imposing predefined safety constraints on the robotic system. The strength of this method lies in allowing the system to perform close to, or at the edge of the constraints' boundaries. In contrast to other works we consider here perceived safety; the constraints for perceived safety are derived in a competitive pHRI experiment. The perceived safety and comfort of the proposed approach is then evaluated with a second, game-like pHRI experiment.
\end{abstract}

\section{INTRODUCTION}

Industrial robots' safety standards are mainly based on isolation of human's and robot's workspaces from each other. However, in physical-human robot interaction (pHRI) autonomous robots need to operate not only in close vicinity of humans but even in direct contact with them [1]. According to Asimov's $1^{\text {st }}$ law, an essential requirement for coexistence of humans and robots is ensuring that the robot would not harm humans (highest priority), the environment, or itself. Therefore, In order to introduce robotic systems into human's daily life, a crucial step is development of a mechanism for pHRI that ensures the human's safety [2], [3], [4]. Two main issues need to be tackled for safe pHRI, namely: passive safety, which is safe mechanical design, and active safety, which results from safe planning and control. The latter's responsibility is to prevent a collision or reduce the effect of collision on the human [5].

A substantial body of work focuses on the control of the impact force of robotic manipulators [6], [7], [8], leading to different force control schemes such as impedance control [9]. Such reactive force control schemes are suitable to reduce the impact of a collision with a human. Although, in order to avoid collisions, a suitable motion planning scheme is needed to change the nominal path to a collisionfree path [3], [5], [10], [11], [12]. pHRI requires the robot to operate in uncertain dynamic environments, the robot needs to avoid any potential unwanted collision online at minimum computational expenses. Finding a collision free path online is generally computationally complex and it often results in a very conservative behavior [5]. When

\footnotetext{
${ }^{1}$ Institue of Automatic Control Engineering, Faculty of Electrical Engineering and Computer Science, Technische Universität München, 80290 Munich, Germany $\{$ sholeh.norouzzadeh,hirche@tum.de\}

2 Experimental Psychology, Ludwig-Maximilians Universität, Munich, Germany $\{$ tamara.lorenz@lmu.de $\}$
}

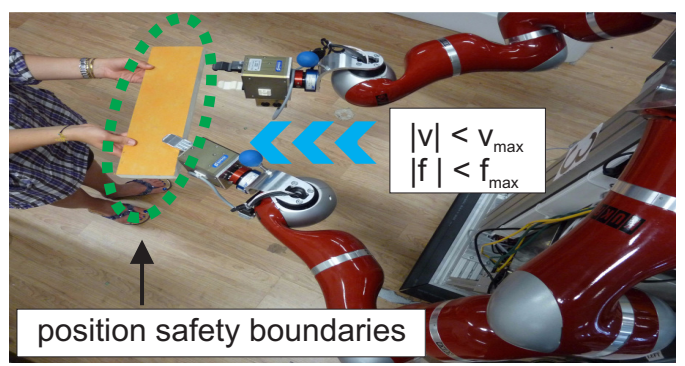

Fig. 1. The force, velocity, and the position of manipulator should be lower than human acceptance threshold for safe interaction.

direct contact is desired as in pHRI, for ensuring safety, the states, such as velocity should be constrained. To reduce the probability of collision with human, some works use a high level safety controller to minimize this probability using an optimal control scheme [2], [5]. The optimal control's cost function represents either the level of injury in case of collision, or the severity of collision based on the point of contact [2], [3], [5]. Although, optimal control methods are used for ensuring high level safety in pHRI, they compute the optimal control signal for all the states offline. This is not suitable for uncertain dynamic environments, because building a look up table for such environment is computationally expensive. Many of the existing methods operate far from the constraints resulting in conservative behavior [13], [14]. Operating far from constraints is a major drawback of optimal control methods. Besides optimal control methods, invariance control is proposed to keep the robot within a predefined safe zone [15]. So far the method works only with $6 \mathrm{D}$ workspace constraints, but neither forces nor velocities are included.

In this paper, we investigate the safety problem from a different perspective. We aim to establish a pHRI which is perceived safe by the human partner. Perceived safety is important for building trust in the human interaction partner and also it is a sufficient condition for safety. The goal is to provide maximal robot task performance while satisfying the constraints on the position, velocity, and force induced by the perceived safety at low computational cost, see also Fig. 1 for an illustration. The proposed method serves between classical control methods and global offline trajectory planners and completes the safety architecture to ensure safety without compromising the current task performance. To this end we consider model predictive control, MPC, which is an online optimal control method solved for the current state as initial state of the controlled system [13], [14]. We design our human-centered MPC using the safety constraints derived from a psychological identification experiment. In order to 


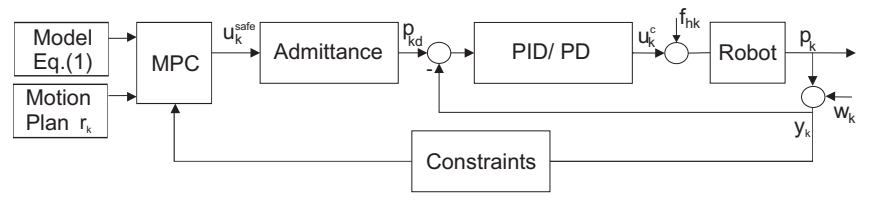

Fig. 2. Schematic of control loop designed to ensure safety in pHRI.

evaluate the perceived safety and comfort resulting from human-robot interaction, we perform a game-like competitive experiment with a two-degrees-of-freedom (2 DOF) haptic device.

This paper is organized as follows: Section II presents the problem definition. Section III reviews the model predictive control scheme. The simulation results are presented in Section IV. The experiments are described and results are discussed in Section V.

\section{Problem Definition}

The goal of this paper is to find a control method to achieve pHRI which is perceived safe and comfortable by the interacting human, while the performance of the robotic manipulator is maximized. We focus here on the most challenging case of pHRI, where the human is in continuous contact with the robot. One crucial question in this respect is how to model the human behavior. Learning methods are successfully employed to predict human intention in pHRI in a probabilistic fashion [1], those approaches are not suitable for absolute safety guarantees.

To avoid this problem, we consider the human's intention as unknown. We consider a problem of controlling the linearized dynamics of a robotic manipulator in the task space, which is linearized by appropriate feedback linearization schemes [1], [2]. The discretized, linear, time invariant model of the manipulator is given by

$$
\begin{aligned}
& \mathbf{x}_{k+1}=A \mathbf{x}_{k}+B_{1} u_{k}+B_{2} f_{h k}, \\
& y_{k}=C \mathbf{x}_{k}+w_{k} .
\end{aligned}
$$

the position and velocity of the manipulator at time $\mathrm{k}$ are represented by $\mathbf{x}_{\mathbf{k}}=\left(p_{k}, v_{k}\right)$. The input $f_{h k}$ is the unknown, unmodeled human force which is exerted on the end-effector of the robot. No measurement is available for this force, we measure the mutual force of the manipulator and the human at the contact point. The measured position of the endeffector is $y_{k}$, and $w_{k}$ is zero-mean white noise (ZMWN) which represents the measurement noise. The matrices $A, B_{1}$, and $B_{2}$ contain the manipulator dynamics, $C$ is the output matrix which is detailed out in section IV, and the control input is $u_{k}$. The goal is to design the control input $u_{k}=u_{k}^{\text {safe }}$ such that the task performance of the robot is expressed in terms of a cost functional $J(u, k)$ is optimized while constraints $g($.$) on position, velocity, and the exerted force$ are satisfied, $\min _{u} J(u, k)$ s.t $g(\mathbf{x}, u) \leq 0$. To address this problem we propose a model-predictive control scheme. The constraints, which represent the "perceived safety zone" for the human, are derived in a human user study.

\section{Model Predictive CONTROL FOR SAFETy IN PHRI}

To deal with the safety constraints, we use model predictive control scheme [13], [16]. The overall control architec-

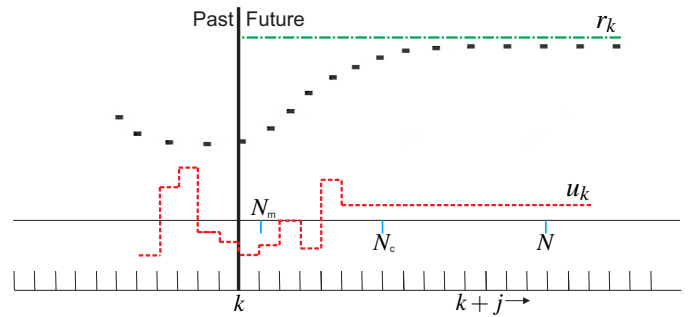

Fig. 3. Receding horizon in MPC.

ture that we propose for ensuring safety in pHRI is shown in Fig. 2. In this architecture we assume that the global motion planner defines the desired trajectory $r_{k}$. Based on this desired trajectory and the constraints, the MPC block responsibility is to compute the desired safe force $u_{k}^{\text {safe }}$. The admittance block, which is added to reduce the impact of collisions, transforms this force into desired position, $p_{k d}$. The error $p_{k d}-p_{k}$ is fed into the classical position control block with high gain to derive the control signal $u_{k}^{c}$. In contact with the human the robot dynamics is driven by the sum of the motor forces $u_{k}$ and the external force $f_{h k}$ applied by the human. The recent measurements are compared to the safety constraints, based on the distance between the measurements and references and with respect to constraints the MPC block takes compensating safe action. The optimization algorithm computes a sequence of future control signals that optimize the cost function, $J$, subject to safety constraints. However, only the first control sample will be implemented. Then the horizon is shifted one sample and the optimization is repeated, see Fig. 3.

\section{A. Generalized Predictive Control Performance Index}

In this paper, we use the generalized predictive control method (GPC) [13], [14], [16], which is the simplest cost function that handles SISO system. We use this cost function for safety in pHRI because we can both punish the position errors and high force changes. The following optimization is done subject to safety constraints, see Fig. 2. Based on the model of Eq. (1) we estimate the position and velocity of the system. Here we assume that the future values of $f_{h k}$ and $u_{k}$ are constant and equal to the previous step, $k-1$. We measure the mutual force, which is $f=u_{k}+f_{h k}$, at the interaction point and we have the $u_{k}$ from previous optimization. Thus, the MPC block computes the optimal control input, $u_{k}^{\text {safe }}$, online based on the prediction $\hat{y}_{k+j \mid k}$ of the output and $\hat{\mathbf{x}}_{k+j \mid k}$ of the state at time $k+j$, assuming the noise $\hat{\mathbf{w}}_{k+j \mid k}=0$ for $j>0$ given the measurements up to time step $k$. Nevertheless, this prediction might be different from real measurements.

$$
\begin{aligned}
& \hat{\mathbf{x}}_{k+j \mid k}=A^{j} \mathbf{x}_{k}+\sum_{i=1}^{j} A^{i-1} B_{1} u_{k+j-i \mid k}+\sum_{i=1}^{j} A^{i-1} B_{2} f_{h k}, \\
& \hat{y}_{k+j \mid k}=C \hat{\mathbf{x}}_{k+j \mid k}+\hat{w}_{k+j \mid k} .
\end{aligned}
$$

We define the predicted control error at time $k+j$ as $\hat{e}_{k+j \mid k}=r_{k+j}-\hat{y}_{k+j \mid k}$. Also, in this paper we assume that the position is the measured output, i.e. $C=\left(\begin{array}{ll}I & 0 \\ 0 & 0\end{array}\right)$, where $I$ is an identity matrix of appropriate dimension. The MPC controller minimizes the following performance index 


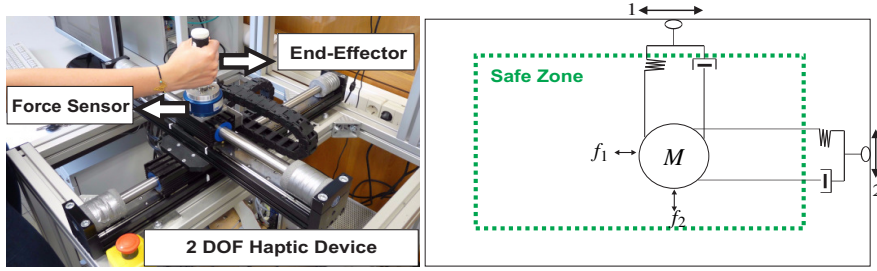

Fig. 4. Schematic of 2 DOF control problem. The arrows show the movement direction. The operation should be within the safe zone shown by the dashed green line.

at time $k$

$$
J(u, k)=\sum_{j=N_{m}}^{N}\left(\hat{e}_{k+j \mid k}\right)^{T}\left(\hat{e}_{k+j \mid k}\right)+\lambda^{2} \sum_{j=1}^{N_{c}} \Delta u_{k+j-1 \mid k}^{T} \Delta u_{k+j-1 \mid k},
$$

subject to

$$
\begin{aligned}
& -\Delta u_{\min }<\Delta u_{k}<\Delta u_{\max } \& \quad-u_{\min }<u_{k}<u_{\max }, \\
& -y_{\min }<\hat{y}_{(k+j)}<y_{\max } .
\end{aligned}
$$

the incremental input signal is $\Delta u=u_{k}-u_{k-1}$, the parameter $N$ is the prediction horizon, $N_{c}$ is the control horizon, $N_{m}$ is the minimum cost horizon, and $\lambda$ is the weighting on the control signal. At each time step the optimization is repeated with new measurements (receding horizon principle). A delay exists between implementing the input and seeing any effect, so $N_{m}>1$. Also, it is assumed that $N_{c} \leq N$, and $\Delta u_{k+j \mid k}=0$ for $j \geq N_{c}+1$, see Fig. 3. It is possible to apply the input signal $u_{k}$ or its increments $\Delta u_{k}$ to the model. In order to achieve a good steady-state behavior, we use an incremental state space model that removes the steady-state tracking errors [13].

\section{B. Optimization and Constraints}

The aim is to optimize the objective function subject to safety constraints (3). The GPC value function that we use is a quadratic value function. As a result, in an unconstrained case, the QP optimization problem that is solved to derive the control input, is convex and convergence to the global optimum is guaranteed. However, when we add the constraints to the optimization problem, on the one hand the problem might be infeasible, on the other hand convexity and smoothness can not be guaranteed everywhere. In this paper, we consider a quadratic cost function subject to linear inequality constraints, which are active on hyperplanes. Consequently, the constrained objective function is a convex surface which is cut off by number of flat surfaces, that keeps the convexity property.

Infeasibility of solution is a major challenge in solving constrained optimization of pHRI problem. In such a case, the quadratic programming solver stops without returning any solution. One option is to use the value $\hat{u}_{k+j+1 \mid k+j-1}$ from the last step of optimization and feed it into the system, but as the predictions are not accurate it can cause an unsafe situation for the human user which is not acceptable. Various approaches have been suggested to deal with this problem like actively managing constraints at each time step, have been proposed in classical MPC literature [13]. In this paper we will not treat the infeasibility problem in depth.

\section{Simulations}

In this section, we use a model of a 2DOF robotic end-effector for running sets of simulations to study the effects of using MPC in the pHRI safety problem, see Fig. 4. The mass, which represents the end-effector, is attached to two decoupled spring damper systems. Each of these systems can only move in one direction, that in total results in 2 DOF. To ensure safety aside from constraining the position, the velocity and the force should also be within the predefined safety constraints. The dynamics of system is decoupled and is represented by a mass-spring-damper system $M \dot{v}+D v+K p=f$, along each dimension where $f \in \mathbb{R}^{p \times 1}$ is the combined force of control input $u$ and the human $f_{h}$ (either $f_{1}$ or $f_{2}$ based on direction, see Fig. 4), $M$ is the virtual mass, $D$ is the damping coefficient, and $K$ is the stiffness. The human force is modeled as an unknown input here, since deriving a detailed model of human behavior is impractical or computationally expensive.

The states of the system are $\mathbf{x}=(p, v)^{T}, \quad \mathbf{x} \in \mathbb{R}^{2 \times 1}$, where $p$ is the position and $v$ is the velocity of the system in either direction of movement. The continuous system is discretized with sampling time $\Delta t$, and the discrete, lineartime-invariant-state-space model is computed as

$$
\begin{gathered}
\left(\begin{array}{c}
p_{k+1} \\
v_{k+1}
\end{array}\right)=\left(\begin{array}{cc}
1 & \Delta t \\
-M^{-1} K \Delta t & 1-M^{-1} D \Delta t
\end{array}\right)\left(\begin{array}{c}
p_{k} \\
v_{k}
\end{array}\right)+\left(\begin{array}{c}
0 \\
M^{-1} \Delta t
\end{array}\right)\left(u_{k}+f_{h k}\right) \\
y_{k}=p_{k}+w_{k}
\end{gathered}
$$

The robot must keep the human safe without any prior knowledge about the human's intention. The only assumption regarding human is that the exerted human force is bounded. Moreover, safety should not compromise the desired behavior of a robot more than necessary. The aim is to compute the optimal control input $u_{k} \in \mathbb{R}^{p \times 1}$ that is able to keep the robot at within the predefined safety zones, while a robot is trying to achieve its own objective.

The parameters of the model and cost function, and the constraints are summarized in Table I. The mass can move in the workspace as long as it stays in the safe zone. In all the experiments the end-effector starts from the workspace center which lies at $(0,0)$. The GPC cost function is optimized at each step to compute the safe optimal force signal. The schematic of the problem is shown in Fig. 4. In the first

TABLE I

OPTIMIZATION AND MODEL PARAMETERS.

\begin{tabular}{|l|l|l|l|l|}
\hline Parameter & $M(K g)$ & $D\left(\frac{N S}{m}\right)$ & $K\left(\frac{N}{m}\right)$ & $\Delta t$ \\
\hline Amount & 40 & 30 & 10 & 0.1 \\
\hline Parameter & $N$ & $N_{c}$ & $N_{m}$ & $\lambda$ \\
\hline Amount & 30 & 20 & 2 & 0.3 \\
\hline Parameter & $\Delta u_{\min }(N)$ & $\Delta u_{\max }(N)$ & $u_{\min }(N)$ & $u_{\max }(N)$ \\
\hline Amount & -2 & 2 & -10 & 10 \\
\hline Parameter & $y_{\min }(m)$ & $y_{\max }(m)$ & $v_{\min }\left(\frac{m}{s}\right)$ & $v_{\max }\left(\frac{m}{s}\right)$ \\
\hline Amount & -6 & 6 & -0.5 & 0.5 \\
\hline
\end{tabular}

simulation, we consider the human is pushing the mass toward the vertical positive direction with a force of $1 \mathrm{~N}$ for 30 steps ${ }^{1}$. The desired reference trajectory is a step

\footnotetext{
${ }^{1}$ Results are reported for one direction and can be used to both directions.
} 


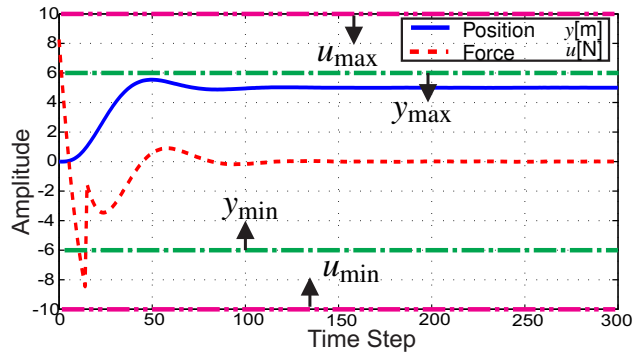

Fig. 5. MPC used as safety control for the 1DOF robotic end-effector.

of size 5. The human force contributes in accomplishing the task. During first few seconds, it helps the human to push the mass in the positive direction, but as it reaches the final position the system disagrees with the human force and tries to stop the motion. Fig. 5 shows the result of using MPC in normal mode. In the next simulation the desired task is similar, but we modify the force safety constraints to $-5 \mathrm{~N} \leq u_{k} \leq 5 \mathrm{~N}$. The result of this simulation is shown in Fig. 6: the force constraint becomes active for 12 steps $(1.2 \mathrm{~s})$, when the force wants to pass the safety limit. The controller keeps the force within the safe boundaries, however, as a result of tightening the force constraint the position settling time is higher. In the third simulation, we

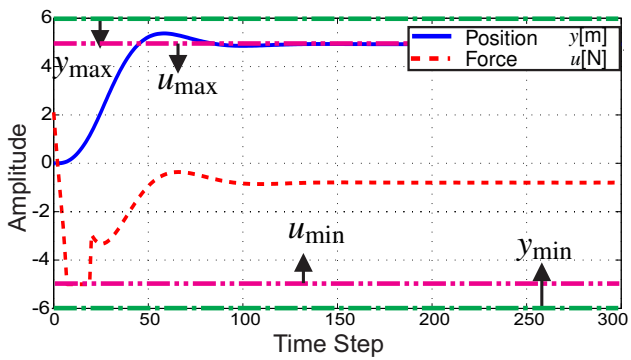

Fig. 6. MPC used as safety control for the 1DOF robotic end-effector with tightened force constraints.

tighten the position safety constraints to $-1 \mathrm{~m} \leq y \leq 6 \mathrm{~m}$, and change the reference trajectory to a step of size -5 . In order to reach this position the robot needs to violate the position safety constraints. The controller's priority is dealing with the exceeding constraint before a catastrophic failure happens. Eventually, the controller keeps the position approximately at -0.5 which is close to the safety bound, but the force peaks are relatively high. These peaks can cause uncomfortable interaction between human and robot. To reduce the force peaks, we increase $\lambda$ to 0.7 and increase the control horizon to 50, respectively. As we can see in Fig. 7, the peak of the force is reduced drastically, but the position reaches the boundary limit which is -1 . This shows the trade off between position tracking error and the desired input force that is always present in this system. Overall, based on the simulations we conclude that MPC can successfully handle the safety constraints. We will validate these results with experiments in the next section.

\section{HUMAN USER STUDY}

\section{A. Experimental Setup}

The haptic interface consists of a 2 DOF linear-actuated device (TrustTube) having a free-spinning handle at the

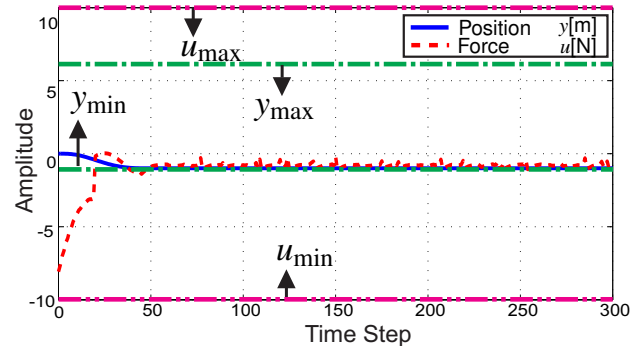

Fig. 7. Tuned MPC used as safety control for the 1DOF robotic end-effector acting in panic mode.

grasping point. Attached to the handle, a force/torque sensor (JR3) measures the human force input. The virtual scene is visually represented on a display placed on top of the interface, see Fig. 4. The control scheme implemented in $M A T L A B /$ Simulink is executed on a personal computer on the Linux/PreemptRT using MATLAB's Real-Time Workshop.

\section{B. Experiment 1: Identification of Comfortable Force Limit}

The capability to interact with humans such that they perceive the interaction as safe although they are unaware of the robot's intention, is necessary for pHRI. The forces that a robot applies when it comes to direct contact with a human should not exceed human capabilities. Therefore, instead of assuming safety constraints as we did in the simulation part, we created an experiment to find out about the human perception of reaction forces applied by a 2 DOF haptic device, Fig. 4. We considered a competitive pHRI task for the experiment because it is the worst case scenario and both partners are trying to follow their own objective. The experiment is designed as a positioning task in which the human has to keep a certain position and the end-effector applies forces in a non-predictable way. The goal of the human is conflicting with the goal of the robotic manipulator.

1) Participants: In total, 14 persons (4 females) aged 26 to 31 years $(M=27.8$ years) participated in the experiment.

2) Setup and Procedure: We defined a simple scenario, where the device is supposed to disturb a human partner with periodic pulses on the two axes, while the human is supposed to stabilize the device in the center point (conflicting goals). The human is instructed to keep the end-effector in the middle and has no knowledge of the manipulator's desired goal. To find the force which is still under human control, we measured the maximum peak of force participants can tolerate while they are still able to keep the device in the middle. We predefine the force exerted by the device to the human in this experiment. In total, three conditions were introduced: (1) weak disturbance force with $4[\mathrm{~N}]$ peaks; (2) stronger disturbance with $8[\mathrm{~N}]$ peaks; (3) high disturbance with $12[\mathrm{~N}]$ peaks. Forces were measured at the contact point to derive the threshold of human tolerance. Each participant was performing each condition once. The conditions were always presented in the order 1-2-3 to give participants the ability to adapt to higher forces as well. After each condition, participants were asked to rate whether they perceived the force as weak, normal, high or too high (not controllable).

3) Results: The average position disturbance of condition (1) was $144.2 \mu \mathrm{m}$, (2) was $4275 \mu \mathrm{m}$ and (3) 


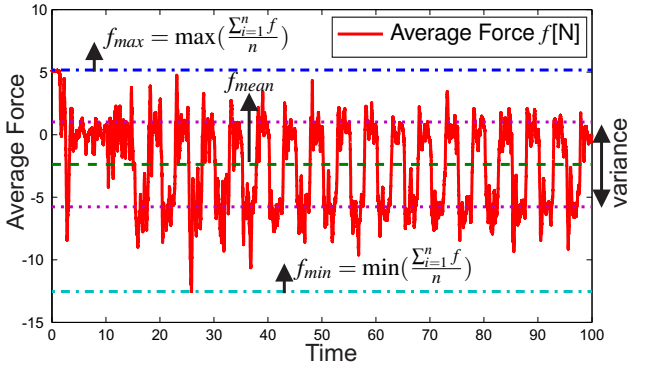

Fig. 8. Measured force averaged over all participants $n=14$.

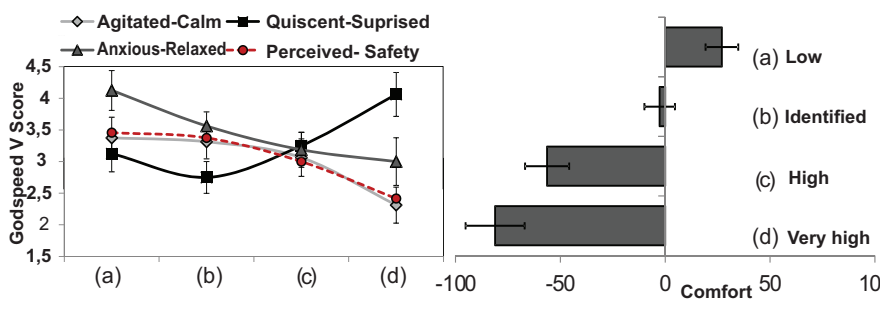

Fig. 9. Average comfort rating per peak force condition (right). Rankings of the Godspeed $V$ questionnaire; the dashed line depicts the cumulated perceived safety (left); error bars depict standard error of the mean.

was $6595 \mu \mathrm{m}$. However, most of the participants were not comfortable when the peak of the force exceeds $4[\mathrm{~N}]$. Already for condition (2) $64 \%$ of participants ranked the force as high. In the following, we therefore only report the result of the first condition with lowest disturbance, see Fig. 8. The maximum measured force in horizontal direction is computed as $\max \left(\left|F_{\max }\right|,\left|F_{\min }\right|\right)$ which is equal to $12.5[\mathrm{~N}]$. We use this maximum as safety boundary for designing the MPC controller for the 2 DOF haptic device. In the same way the maximum acceleration is approximated as $0.3 \frac{\mathrm{m}}{\mathrm{s}^{2}}$ and the maximum velocity equal to $0.6 \frac{\mathrm{m}}{\mathrm{s}}$. These constants and the same model Eq. (4), (5) of simulations discretized with $\Delta t=0.001 \mathrm{~s}$ is used for the second experiment in which we measure the human comfort during interaction with the haptic device using MPC as a safety controller.

\section{Experiment 2: Comfortable Interaction}

Using the identification results of Experiment 1 we designed a second experiment in which the human and the robot have different goals. The objective of this experiment is to gain knowledge about the comfort and the perceived safety participants feel when interacting with the same 2 DOF haptic device, now with MPC as safety control method.

1) Participants: Overall, 16 persons (4 female) took part in this experiment. They were between 25 and 31 years old $(M=27,9$ years $)$ and reported not to suffer any motorical restrictions in their right arm/hand.

2) Setup and Procedure: In order to visualize the task, a circle of targets was presented on the screen above the 2 DOF device, see Fig. 10. Before the start of the experiment, the handle of the 2 DOF device was positioned in the center which matched the blue circle in the center of the circle of targets, see Fig. 10. After reading the instructions, participants were asked to stand in front of the device. Their task was, to grasp the handle and, by pushing the handle into an indicated direction, hit all targets (red circles) one

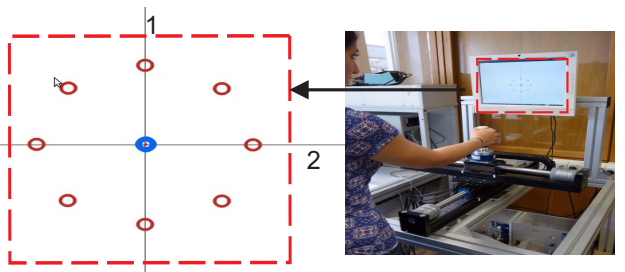

Fig. 10. Experimental Setup: visualization of the game scenario, circle of targets presented on the screen (left). 2 DOF haptic device (right).

after each other. Counter productive to this action, the robot's task was to keep the handle in the middle. Participants were unaware of this intention. Performing the experiment they were only allowed to try and hit each target once by pushing the handle in the indicated direction. The first target was defined by the experimenter and participants were instructed to hit the subsequent targets either in clockwise or counter clockwise order. In total four different levels of peak force were introduced: (a) low, which was defined as half of the identified force $(12.5[\mathrm{~N}] / 2=6.25[\mathrm{~N}])$; (b) identified (12.5 [N]); (c) high, which was defined as double the identified force $(12.5[\mathrm{~N}] * 2=25[\mathrm{~N}])$; and (d) very high $250[\mathrm{~N}]$. For each condition, a full target circle has to be completed. Conditions were assigned randomly as were first targets and hitting order direction. After each condition, participants were asked to fill in a questionnaire regarding their perceived safety during interaction and their rating of comfort. To access the perceived safety we utilized the block $V$ taken from the Godspeed questionnaire in which the participant has to rate his/her impressions on three 5-point semantic differential pairings regarding their impression after interacting with the device [17]. Pairings of the scales are anxious/relaxed, agitated/calm and quiescent/surprised. Note that the pairing quiescent/surprised contributes in a reversed way: being surprised by the reactions of the device causes lower perceived safety. For assessing comfort level, we used the continuum subjective ranking scale introduced by Kong et al. [18]. Participants mark their perceived level of comfort on a line of $10 \mathrm{~cm}$ ranging from very uncomfortable $(-100)$ to very comfortable $(+100)$. Data Analysis was performed in MS Excel and SPSS 19. For all ratings, one-way repeated measures ANOVAs were calculated over force conditions.

3) Results and Discussion: Regarding comfort, the ANOVA revealed a significant main effect between peak forces, $F(3,45)=5.88, p<.01$. Planned comparisons show, that low $(M=27.00)$, identified $(M=-0.64)$ and high force $(M=-56.43)$ were perceived as more comfortable than the very high force $(M=-80.07)$, see Table II. This is not surprising as the very high peak force (d) of $250[\mathrm{~N}]$ did not allow participants to move the handle in any direction. Thus, instead of a compliant device, the robot appears as a solid body. Colliding with a robot like that would definitely imply the risk of injury which is depicted in the high discomfort rating (negative comfort). In contrast, the low force (a) and the identified force (b) were rated significantly better, see Table II, whereby the latter was rated almost at the threshold between comfort and discomfort. Summing up it appears that the low peak force (a) was the most comfortable, the identified peak force (b) was proven to 
TABLE II

PLANNED COMPARISONS

\begin{tabular}{c|cccccc|ccc|cc}
\hline Force & \multicolumn{2}{|c}{ Anxious-Relaxed } & \multicolumn{2}{c|}{ Agitated-Calm } & \multicolumn{2}{|c|}{ Quiescent-Surprised } & \multicolumn{3}{|c}{ Perceived Safety } & \multicolumn{3}{|c}{ Comfort } \\
& $F(1,15)$ & $p$ & $F(1,15)$ & $p$ & $F(1,15)$ & $p$ & $F(3,45)$ & $p$ & $F(3,39)$ & $p$ \\
\hline (a)-(b) & 2.93 & $n . s$. & 0.041 & $n . s$. & 1.22 & $n . s$. & 0.12 & $n . s$. & 3.09 & $n . s$. \\
(a)-(c) & 12.45 & $<0.01$ & 0.63 & $n . s$. & 0.11 & $n . s$. & 2.77 & $n . s$. & 30.43 & $<0.001$ \\
(a)-(d) & 11.80 & $<0.01$ & 8.23 & $<0.05$ & 5.42 & $<0.05$ & 10.48 & $<0.01$ & 33.44 & $<0.001$ \\
(b)-(c) & 2.14 & $n . s$. & 0.79 & $n . s$. & 3.00 & $n . s$. & 2.99 & $n . s$. & 15.34 & $<0.01$ \\
(b)-(d) & 1.90 & $n . s$. & 7.06 & $<0.05$ & 7.74 & $<0.05$ & 8.79 & $=0.01$ & 26.85 & $<0.001$ \\
(c)-(d) & 0.28 & n.s. & 4.09 & $=0.06$ & 3.56 & $=0.08$ & 5.00 & $<0.05$ & 6.90 & $<0.05$
\end{tabular}

be the comfort threshold, while the higher peak forces (c) and (d) were rated as seriously uncomfortable and are thus not applicable if the interaction partners human and robot are unaware of each other's intention. The ANOVA on the cumulated perceived safety shows that peak forces also caused a difference in perceived safety, $F(3,45)=5.88, p<.01$. Numerically, the low force $(M=3.46)$ caused a higher perceived safety compared to the identified force $(M=3.38)$ and the high force $(M=3.00)$, see V-C.2. Although this result did not reach significance, the low and the identified peak force resulted in a perceived safety above the middle ranking of 3.00, while the high force was rated to be at this threshold. The very high peak force was rated to have a significantly lower perceived safety compared to all the others, see Table II. Interestingly, the results for the pairing quiescent/surprised shows the lowest rating at the identified peak force. This might indicate that the low force, although being most comfortable, was also not expected. It might differ from previous experience participants could have in contact with comparable devices. Note that for the applied computation of perceived safety, higher surprise - whether positive or negative - is treated as unwanted and thus scales down perceived safety.

The final argument for concluding the experiments is that safety and efficient performance are two essential characteristics for successful pHRI. In this paper we develop a control architecture that keeps the human safe while trying to maximize the performance. By using the MPC scheme as safety controller, the manipulator performs within the predefined safety boundaries. Moreover, the designed controller's perceived comfort is at the threshold, which means higher forces than the identified one can cause uncomfortable pHRI. If a feeling of comfort should be created, peak forces should be scaled down. However, if the performance of the manipulator is important, then the designed controller will achieve the highest comfortable force. Overall, we conclude that the designed MPC controller has the best trade off between efficiency and safety among the four tested conditions.

\section{CONCLUSIONS}

In this paper, we present a control architecture to ensure perceived safety in physical human-robot interaction, where the human is in continuous contact with the robot. It involves an an online optimal control, which imposes constraints on interaction forces, position, and velocity of the robotic system. The suggested model-predictive control approach enables the robot to optimize its desired performance as long as the perceived safety and comfort of the interacting human are not endangered. The interaction force constraints for perceived safety are derived in a human user study in a 2 DOF haptic virtual environment. In a second human user study on pHRI it is shown, that indeed the human feels safer and more comfortable when the robot is operated under MPC with the derived force constraints.

\section{ACKNOWLEDGEMENTS}

This work is supported within the DFG excellence initiative research cluster Cognition for Technical Systems CoTeSys (www.cotesys.org).

\section{REFERENCES}

[1] J.R. Medina, M. Lawitzky, A. Mörtl, D. Lee and S. Hirche, An Experience-Driven Robotic Assistant Acquiring Human Knowledge to Improve Haptic Cooperation. International Conference on Intelligent Robots and Systems (IROS), pp. 2416-2422, 2011.

[2] S. Haddadin, Towards Safe Robots: Approaching Asimovs 1st Law, Ph.D. Dissertation, 2011.

[3] A. De Santis, B. Siciliano, A. De Luca and A. Bicchi, An atlas of physical human-robot interaction. Mechanism and Machine Theory, vol. 43 , no. 3 , pp. 253-270, 2008.

[4] A. J. Bearveldt, Cooperation between Man and Robot: Interface and Safety, IEEE International Workshop on Robot Human Communication, pp. 183-187,1993.

[5] D. Kulic and E.A. Croft, Real-time safety for human-robot interaction. Robotics and Autonomous Systems, vol. 54, no. 1, pp.1-12, 2006.

[6] J.K. Salisbury, Active stiffness control of manipulator in Cartesian coordinates. IEEE Conference on Decision and Control, pp. 249-256, 1996.

[7] R. Paul and B. Shimano, Compliance and control. Joint Automatic Control Conference (JACC), pp. 694-699, 1976.

[8] J.J Craig and M.H. Raibert, A systematic method of hybrid position/force control of a manipulator. Proceedings in Computer Software and the IEEE Computer Society's Third International Applications Conference, pp. 446-51, 1979.

[9] N. Hogan, and P. M. Grant, Impedance control- An approach to manipulation. ASME Transactions Journal of Dynamic Systems and Measurement Control B, vol. 107, pp. 1-24, 1985.

[10] H. Ding, K. Wijaya, G. Reissig, and O. Stursberg, Optimizing Motion of Robotic Manipulators in Interaction with Human Operators, International Conference on Intelligent Robotics and Applications, pp.520$531,2011$.

[11] K. Ikuta and M. Nokata, Safety Evaluation Method of Design and Control for Human-Care Robots, The International Journal of Robotics Research, vol.22, no.7, pp. 281-297, 2003.

[12] O. Khatib, Real-time obstacle avoidance for manipulators and mobile robots, The International Journal of Robotics Research, vol.5, pp. 9098, 1985.

[13] J.M. Maciejowski, Predictive Control with Constraints. Prentice Hall, 2002.

[14] D.Q Mayne, J.B Rawlings, C.V Rao and P.O.M Scokaert, Constrained Model Predictive Control: Stability and Optimality, Automatica, vol.36, pp. 789-814, 2000.

[15] M. Kimmel, M. Lawitzky and S. Hirche, 6D Workspace Constraints for Physical Human-Robot Interaction using Invariance Control with Chattering Reduction, International Conference on Intelligent Robots and Systems (IROS), 2012.

[16] B. De Schutter and T.J.J. van den Boom , Model predictive control for max-plus-linear discrete event systems , Automatica, vol.37, no.7, pp. 1049-1056, July 2001.

[17] C. Bartneck and D. Kulic, Measuring the anthropomorphism, animacy, likeability, perceived intelligence and perceived safety of robots, Metrics for HRI Workshop,vol. 471, pp. 37-44, 2008. 
[18] Y.-K. Kong, D.-M. Kim, K.-S. Lee, and M.-C. Jung, Comparison of comfort, discomfort, and continuum ratings of force levels and hand regions during gripping exertions, Applied Ergonomics, vol.43, pp. 283-289, 2012. 